\title{
On Optimization of the V-BLAST Algorithm
}

\author{
Victoria Kostina, Sergey Loyka \\ School of Information Technology and Engineering University of Ottawa, \\ 161 Louis Pasteur, Ottawa, Ontario, Canada, K1N 6N5 \\ E-mail: sergey.loyka@ieee.org
}

\begin{abstract}
Optimum power allocation for the V-BLAST algorithm, which is based on various criteria (average and instantaneous block and total error rates (BLER and TBER)), is considered. Closed-form expressions are derived for high-SNR case in a Rayleigh fading channel. It is demonstrated that, in that case, the optimization "on average" is almost identical to the instantaneous one (while the former requires only the feedback "on average", the latter requires instantaneous feedback and hence is of higher complexity). The BLER and TBER optimization criteria result in the same performance. Power optimization (of un-ordered BLAST) and optimal ordering result in the same performance improvement at high SNR.
\end{abstract}

\section{INTRODUCTION}

The BLAST algorithm originally proposed by Foschini [1][2] has attracted significant attention in recent years as comparatively simple yet optimum solution (recall that the MMSE BLAST achieves the full MIMO capacity [1]). The algorithm however suffers from several drawbacks. Its computational complexity is still large for many applications, and also the algorithm BER performance is degraded by the effect of error propagation. Thus, some modifications have been proposed to improve the algorithm in these directions [3][4].

In the present paper, we consider an optimum transmit power allocation to improve the BLAST error rate performance using various optimization criteria from a unified perspective. The optimization criteria considered include instantaneous and average block error rate (BLER) and total error rate (TBER). It is shown that at high SNR mode, the optimization "on average" is almost identical (in terms of the average BER) to the instantaneous one for a Rayleigh fading channel, when either BLER or TBER used as the optimization criteria. Since the optimization "on average" does not require instantaneous feedback, its implementation complexity is much less as compared to the instantaneous one, especially in a fast-fading channel.

Our optimization results are based on recent analytical performance evaluation of the BLAST [5][6][7], which allows us to derive compact closed-from expressions at high-SNR mode and also to prove the uniqueness of the solution (which facilitates the use of numerical techniques). A generic upper bound on the SNR gain of the optimization is derived. By considering the power-optimized BLAST without optimal ordering and comparing it to the unoptimized BLAST with the ordering, it is demonstrated that they have the same BER performance at high SNR. Hence, optimum power allocation can be used instead of the ordering, with much smaller complexity penalty.

\section{System MOdEL AND THE V-BLAST AlgORITHM}

The following standard baseband MIMO system model is adopted in the present paper:

$$
\mathbf{y}=\mathbf{H x}+\boldsymbol{\xi}=\sum_{i=1}^{m} \mathbf{h}_{i} x_{i}+\xi
$$

where $\mathbf{x}=\left[x_{1}, x_{2}, \ldots x_{m}\right]^{T}$ and $\mathbf{y}=\left[y_{1}, y_{2}, \ldots y_{m}\right]^{T}$ are the $\mathrm{Tx}$ and $\mathrm{Rx}$ vectors correspondingly, $\mathbf{H}=\left[\mathbf{h}_{1}, \mathbf{h}_{2}, \ldots \mathbf{h}_{m}\right]$ is the $n \times m$ channel matrix, i.e. the matrix of the complex channel gains between each Tx and each $\mathrm{Rx}$ antenna, $\mathbf{h}_{i}$ is the $\mathrm{i}$-th column of $\mathbf{H}, n$ is the number of Rx antennas, $m$ is the number of Tx antennas, $n \geq m$, and $\xi$ is the additive white Gaussian noise (AWGN), which is assumed to be $\mathcal{C N}\left(0, \sigma_{0}^{2} \mathbf{I}\right)$, i.e. independent and identically distributed (i.i.d.) in each branch. Additionally, we adopt the same basic assumptions as in [5][7] (the channel is i.i.d. Rayleigh fading (the components of $\mathbf{H}$ are $\mathcal{C N}(0, \mathbf{I}))$, quasistatic, frequency independent; the $\mathrm{Tx}$ signals, noise and channel gains are independent of each other; perfect channel knowledge is available at the receiver; there is no performance degradation due to synchronization and timing errors).

The detection of a Tx symbol in the V-BLAST algorithm proceeds in steps and includes 3 major procedures at each step: 1) interference cancellation from already detected symbols, 2) interference nulling from yet-to-be-detected symbols, 3) optimal ordering (based on after-detection SNR) (a more detailed description of the algorithm can be found elsewhere [1][2] and is omitted here). Since the optimal ordering procedure has a significant computational complexity and hence is one of the major obstacles to costefficient implementation (and also is very challenging for analytical analysis), we exclude it from the algorithm and further demonstrate that an optimum per-stream power allocation at the Tx (based on the average BER) allows to achieve the same result without high complexity penalty.

After the interference cancellation and nulling, the receiver forms the following decision variable at step $i$ :

$$
r_{i}=\mathbf{w}_{i}^{+} \mathbf{h}_{i} x_{i}+\mathbf{w}_{i}^{+} \sum_{j=1}^{i-1} \mathbf{h}_{j} \Delta x_{j}+\mathbf{w}_{i}^{+} \xi
$$

where $^{+}$denotes Hermitian conjugate, $\mathbf{w}_{i}=\mathbf{h}_{i \perp} /\left|\mathbf{h}_{i \perp}\right|$ are the optimum combining weights that completely eliminate the inter-stream interference (ISI) from yet-to-be-detected symbols and maximize the output SNR, $\mathbf{h}_{i \perp}=\mathbf{P}_{i} \mathbf{h}_{i}, \mathbf{P}_{i}$ is the projection matrix on the sub-space orthogonal to that spanned by $\quad\left\{\mathbf{h}_{i+1} \mathbf{h}_{i+2} \ldots \mathbf{h}_{m}\right\}: \quad \mathbf{P}_{i}=\mathbf{I}-\mathbf{H}_{i}\left(\mathbf{H}_{i}^{+} \mathbf{H}_{i}\right)^{-1} \mathbf{H}_{i}^{+}, \quad$ where $\mathbf{H}_{i}=\left[\mathbf{h}_{i+1} \mathbf{h}_{i+2} \ldots \mathbf{h}_{m}\right], \quad$ and $\quad \Delta x_{j}=\hat{x}_{j}-x_{j} \quad$ represents demodulation error at step $j$, with $\hat{x}_{j}=D^{-1}\left\{r_{j}\right\}$ being the demodulated symbol [6][7]. Based on (2), an exact BER analysis is possible in closed form [6][7]. We outline below the major results of this analysis, which further serve as a tool for optimization.

Noise and error independence: It can be shown [6][7] that the optimum weights are orthogonal, 
$\mathbf{w}_{i}^{+} \mathbf{w}_{j}=\mathbf{h}_{i}^{+} \mathbf{P}_{i} \mathbf{P}_{j} \mathbf{h}_{j}=0 \forall i \neq j$. Hence, for given channel $\mathbf{H}$, the after-combining noises at different steps are independent of each other,

$$
\left\langle\xi_{p, i}{ }^{*} \xi_{p, j}\right\rangle=\sigma_{0}^{2} \mathbf{w}_{i}^{+} \mathbf{w}_{j}=\sigma_{0}^{2} \delta_{i j} ; \xi_{p} \sim \mathcal{C N}\left(0, \sigma_{0}^{2} \mathbf{I}\right)
$$

where $\xi_{p, i}=\mathbf{w}_{i}^{+} \boldsymbol{\xi}$, and $\sigma_{0}^{2}$ is the noise variance (note that noise independence follows from zero correlation as the noise is Gaussian). Hence, the demodulation errors are independent too. This facilitates the error rate analysis.

Instantaneous error rates: The block error rate (BLER), which is a probability to have at least one error in the demodulated Tx vector, can be expressed as

$$
P_{B}=1-\prod_{i=1}^{m}\left(1-P_{e}\left(\gamma_{i}\right)\right) \leq \sum_{i=1}^{m} P_{e}\left(\gamma_{i}\right)
$$

where $P_{e}\left(\gamma_{i}\right)$ is the (instantaneous, i.e. for given channel) conditional (no errors at the previous steps) error rate at step i, $\gamma_{i}=\left|\mathbf{h}_{i \perp}\right|^{2} / \sigma_{0}^{2}$ is the SNR (assuming unit power constellation). Evaluation of the BLER is comparatively simple as it is independent of the error propagation (and hence its quantification is not required). The (instantaneous) total error rate (TBER) $P_{e t}$, i.e. the error rate at the output stream to which all the streams are merged after demodulation, can be bounded using the BLER,

$$
P_{B} / m \leq P_{e t}=\frac{1}{m} \sum_{i=1}^{m} P_{u i} \leq P_{B}
$$

where $P_{u i}$ is the unconditional (includes the error propagation from previous steps) error rate at step $i$.

Average error rates: It can be shown [5] that, in i.i.d. Rayleigh fading channels, $\gamma_{i} \sim \chi_{2(n-m+i)}^{2}$, where " $\sim$ " means "equal in distribution", and are independent of each other. This independence facilitates the evaluation of the average BLER $\bar{P}_{B}$,

$$
\bar{P}_{B}=1-\prod_{i=1}^{m}\left(1-\bar{P}_{e i}\right)
$$

where $\bar{P}_{e i}=\bar{P}_{(n-m+i)}^{M R C}$ is the average conditional error rate at step $\mathrm{i}$, and the average error rate with $(\mathrm{n}-\mathrm{m}+\mathrm{i})$ order maximum ratio combining (MRC) is $\bar{P}_{(n-m+i)}^{M R C}$, which is known for many modulation formats in closed form. For large average SNR $\left(\gamma_{0}=1 / \sigma_{0}^{2}>>1\right), \bar{P}_{e 1}>>\bar{P}_{e 2}>>\ldots>>\bar{P}_{e m}$ due to increasing diversity order with step number, i.e. the $1^{\text {st }}$ step error rate dominates. Thus,

$$
\overline{P_{B}} \approx \overline{P_{e 1}} \text { for } \gamma_{0}>>1
$$

Average unconditional BER for BPSK: In the case of BPSK modulation, an exact expression for the average unconditional BER at each step and hence for the average TBER can be obtained [6][7]. For simplicity and due to the page limit, we consider further the case of $m=2$; the analysis extends also to $m>2$ [7]. By observing that the last two terms in (2) represent "total noise" (the AWGN plus ISI) that is Gaussian, whose variance is $\sigma_{0}^{2}+\left|\Delta x_{1}\right|^{2}$, the unconditional (taking into account the error propagation from step 1) BER at step 2 for given $\mathbf{h}_{2}$ (but averaged out over $\mathbf{h}_{1}$ ) is

$$
P_{u 2}\left(\mathbf{h}_{2}\right)=Q\left(\sqrt{2 \gamma_{2}}\right)\left(1-\bar{P}_{e 1}\right)+Q\left(\sqrt{\frac{2 \mathbf{h}_{2}^{+} \mathbf{h}_{2}}{\sigma_{0}^{2}+4}}\right) \bar{P}_{e 1}
$$

where $Q(\cdot)$ is the well-known Q-function, and the average BER at step 1 is

$$
\bar{P}_{e 1}=\bar{P}_{(n-1)}^{M R C}=\left[\frac{1-\mu}{2}\right]^{n-1} \sum_{k=0}^{n-2} C_{n-2+k}^{k}\left[\frac{1+\mu}{2}\right]^{k}, \mu=\sqrt{\frac{\gamma_{0}}{1+\gamma_{0}}}
$$

where $C_{n}^{k}=n ! /(k !(n-k) !)$ are the binomial coefficients. Averaging out (8) over $\mathbf{h}_{2}$, one obtains:

$$
\bar{P}_{u 2}=\bar{P}_{e 2}\left(1-\bar{P}_{e 1}\right)+\bar{P}_{21} \bar{P}_{e 1}
$$

where $\bar{P}_{e 2}=\bar{P}_{(n)}^{M R C}$, and $\bar{P}_{21}=\bar{P}_{(n)}^{M R C}\left(1 /\left(\sigma_{0}^{2}+4\right)\right)$ is the average probability of error propagation. The average TBER and BLER can be immediately evaluated using (5)-(10). Large SNR approximations for $n=2$ are especially simple,

$$
\begin{aligned}
& \bar{P}_{e 1} \approx \frac{1}{4 \gamma_{0}}, \bar{P}_{e 2} \approx \frac{3}{16 \gamma_{0}^{2}}, \bar{P}_{21} \approx \frac{1}{5}, \bar{P}_{u 2} \approx \frac{1}{5} \bar{P}_{e 1} \approx \frac{1}{20 \gamma_{0}} \\
& \bar{P}_{e t} \approx \frac{\bar{P}_{e 1}}{2}\left(1+\bar{P}_{21}\right) \approx \frac{1}{7 \gamma_{0}}
\end{aligned}
$$

By comparing $\bar{P}_{e t}$ in (11) to that without error propagation, $\vec{P}_{e t} \approx \bar{P}_{e 1} / 2 \approx 1 /\left(8 \gamma_{0}\right)$, one concludes that the effect of error propagation is to increase the average TBER by $14 \%$, i.e. not catastrophic at all. However, as the comparison of $\bar{P}_{e 2}$ and $\bar{P}_{u 2}$ demonstrates, the error propagation has a profound effect on the $2^{\text {nd }}$ step BER (reducing the diversity order from 2 to 1 ).

\section{OPTIMUM POWER AllocAtion}

Under the total Tx power constraint, individual (per Tx or stream) powers can be optimally allocated in such a way as to minimize the TBER or the BLER, either instantaneous or average. While the instantaneous (i.e. for each channel realization) power allocation requires an instantaneous feedback channel (to supply the Tx end with the optimum allocation for each channel realization), the average power allocation does not require instantaneous feedback (only the average SNR needs to be known at the Tx end) and hence does not incur significant penalty in complexity.

To account for unequal $\mathrm{Tx}$ power distribution, let us introduce the power allocation vector $\boldsymbol{\alpha}=\left[\alpha_{1}, \alpha_{2}, \ldots \alpha_{m}\right]$. The total power constraint is

$$
\sum_{i=1}^{m} \alpha_{i}=m
$$

and uniform (unoptimized) power allocation considered above corresponds to $\alpha_{1}=\alpha_{2}=\ldots=\alpha_{m}=1$. The analysis in Section II has to be modified by introducing new step SNRs $\gamma_{i}^{\prime}=\alpha_{i} \gamma_{i}$ so that

$$
P_{B}(\boldsymbol{\alpha})=1-\prod_{i=1}^{m}\left(1-P_{e}\left(\gamma_{i}^{\prime}\right)\right)
$$

where we have explicitly indicated the BLER as a function of $\boldsymbol{\alpha}$ (it is of course a function of $\gamma_{0}$ as well). Similarly, $P_{e t}=P_{e t}(\boldsymbol{\alpha}), P_{u i}=P_{u i}\left(\alpha_{1} \ldots \alpha_{i}\right)$, and the same relations hold true for the average error rates. Eq. (9) ${ }_{M R C}^{\text {is modified by the }}$ substitution $\quad \gamma_{0} \rightarrow \alpha_{1} \gamma_{0}: \quad \bar{P}_{e 1}=\bar{P}_{(n-1)}^{M R C}\left(\alpha_{1} \gamma_{0}\right), \quad$ and (10) is modified to

$$
\bar{P}_{u 2}\left(\alpha_{1}, \alpha_{2}\right)=\bar{P}_{e 2}\left(\alpha_{2}\right)\left(1-\bar{P}_{e 1}\left(\alpha_{1}\right)\right)+\bar{P}_{21}\left(\alpha_{1}, \alpha_{2}\right) \bar{P}_{e 1}\left(\alpha_{1}\right)
$$

where $\bar{P}_{21}\left(\alpha_{1}\right)=\bar{P}_{(n)}^{M R C}\left(\alpha_{2} /\left(\sigma_{0}^{2}+4 \alpha_{1}\right)\right), \bar{P}_{e 2}=\bar{P}_{(n)}^{M R C}\left(\alpha_{2} \gamma_{0}\right)$. Eq. (11) is also modified in the same way. 
Optimum power allocation using the average BLER: For this optimization, the average BLER is minimized under the constraint (12). Using the Lagrange multiplier technique for constrained optimization with the Lagrangian

$$
L(\boldsymbol{\alpha})=\bar{P}_{B}(\boldsymbol{\alpha})-\lambda\left(\sum_{i=1}^{m} \alpha_{i}-m\right),
$$

the optimum $\boldsymbol{\alpha}$ are found from

$$
\partial L(\boldsymbol{\alpha}) / \partial \alpha_{i}=\partial \bar{P}_{B}(\boldsymbol{\alpha}) / \partial \alpha_{i}-\lambda=0, i=1 \ldots m,
$$

where $\lambda$ is the Lagrange multiplier, which is found from the constraint (12) (i.e., (16) and (12) are considered together as a system of equations).

Uniqueness of the solution: We first show that (15) has a unique solution, which is a minimum (in terms of $\bar{P}_{B}(\boldsymbol{\alpha})$ ), for BPSK modulation. Indeed, let us prove the following Lemma 1: $z(\boldsymbol{\alpha})=\sum_{i=1}^{m} \log \left(1-\bar{P}_{e i}\left(\alpha_{i}\right)\right)$ is concave in $\boldsymbol{\alpha}$ for BPSK modulation. Proof: Consider the instantaneous BER of BPSK $P_{e}(\gamma)=Q(\sqrt{2 \gamma})$; it can be verified by direct computation that

$$
d^{2} P_{e}(\gamma) / d \gamma^{2}>0
$$

which is a sufficient condition for $P_{e}(\gamma)$ to be convex [8]. Since the average BER is obtained by integration of $P_{e}(\gamma)$ with non-negative weight function (the pdf of $\gamma$ ), it follows [8] that $\bar{P}_{e}\left(\gamma_{0}\right)$ is also convex for any diversity order and any combining technique, including the MRC. Thus, $\bar{P}_{e i}\left(\underline{\alpha}_{i}\right)=\bar{P}_{(n-m+i)}\left(\alpha_{i} \gamma_{0}\right)$ is convex and, consequently, $\left(1-\bar{P}_{e i}\left(\alpha_{i}\right)\right)$ is concave. Since log of a concave function is concave [8], $\log \left(1-\bar{P}_{e i}\left(\alpha_{i}\right)\right)$ is concave and, consequently, $z(\boldsymbol{\alpha})$ is also concave Q.E.D. As a concave function, $z(\boldsymbol{\alpha})$ has a unique maximum under the constraint (12) [8], which corresponds to the unique minimum of $\bar{P}_{B}(\boldsymbol{\alpha})$ since $z(\boldsymbol{\alpha})=\log \left(1-\bar{P}_{B}(\boldsymbol{\alpha})\right)$ and $\log$ is a unique monotonically increasing function. We would like to note that this uniqueness result extends to any diversity combining technique (and not only the MRC), and also to any modulation format whose instantaneous BER can be expressed as either $a Q(\sqrt{b \gamma})$ or $a \cdot \exp (-b \gamma)$ (or any linear combination of those with non-negative coefficients) for some constants $a$ and $b$ (and can be also applied to symbol rather than bit error rate).

Using a numerical algorithm, the (globally) optimum power allocation can be found. The uniqueness of the solution facilitates numerical evaluation as there is only one global minimum. For high SNR, (13) is approximated as

$$
P_{B}(\boldsymbol{\alpha}) \approx \sum_{i=1}^{m} \frac{C_{2 i-1}^{i}}{\left(4 \alpha_{i} \gamma_{0}\right)^{n-m+i}}
$$

and a compact and accurate analytical solution to (16) can be obtained (using the Newton-Raphson method; the details are omitted due to the page limit), which is especially simple (and insightful) for $m=2$,

$$
\alpha_{1} \approx 2\left(1-\frac{c}{(n+1) \sqrt[n+1]{\gamma_{0}}}\right)^{n+1}, \alpha_{2}=2-\alpha_{1}, c=\sqrt[n+1]{\frac{3 n}{8(n-1)}}
$$

Fig. 1 compares the approximate solution above with the accurate numerical solution. Clearly, the approximate solution is accurate for $\gamma_{0} \geq 10 \mathrm{~dB}$. Additionally, since the average BLER (and also the TBER) is not very sensitive to small variations in $\boldsymbol{\alpha}$, the approximate solution results in almost the same average BLER (TBER) as the accurate numerical one also for $\gamma_{0}<10 \mathrm{~dB}$. Thus, (19) can be used for the whole range of $\gamma_{0}$ without significant performance degradation.

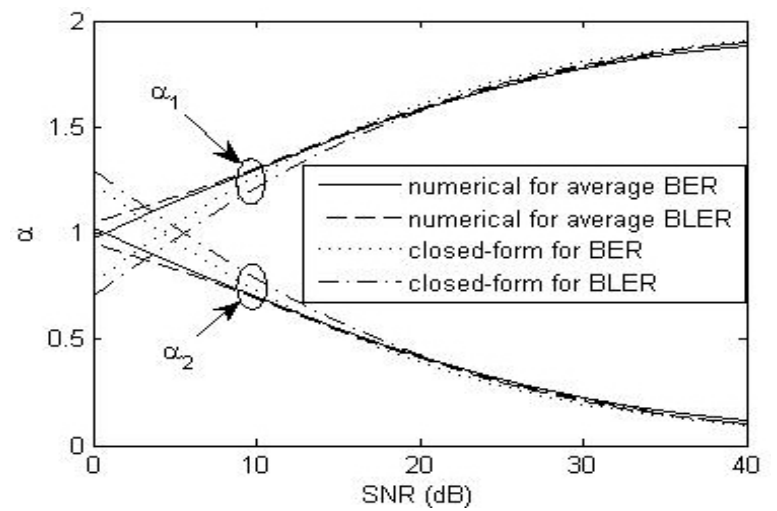

Fig. 1. Optimum power allocation for $2 \times 2$ V-BLAST with BPSK modulation for various optimization strategies.

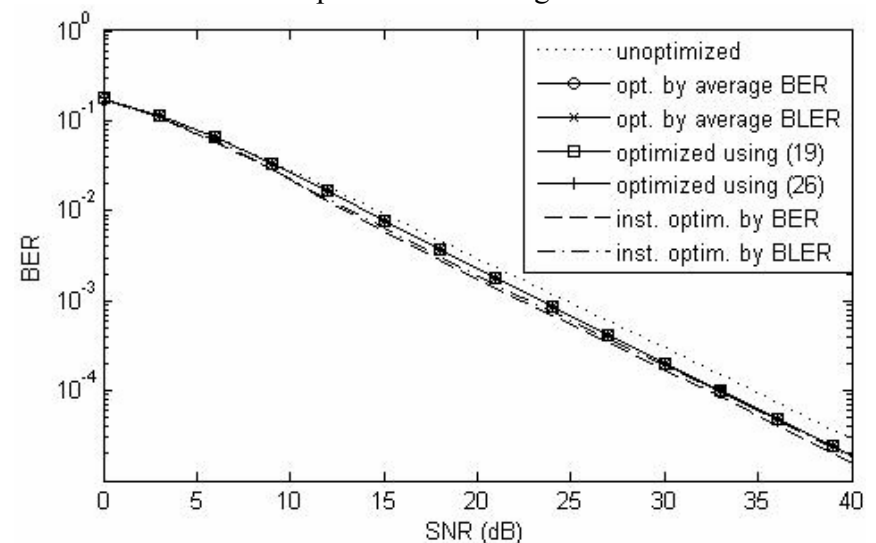

Fig. 2. Average TBER of $2 \times 2$ V-BLAST with BPSK modulation for various optimization strategies.

It follows from (19) that $\alpha_{1} \rightarrow 2$ (i.e. almost all the power goes to the $\left.1^{\text {st }} \mathrm{Tx}\right)$ as $\gamma_{0} \rightarrow \infty$, and $\alpha_{1}$ is quite close to 2 for finite $\gamma_{0}$. Referring to (11), this is explained by the fact that $1^{\text {st }}$ step has lowest diversity order $(n-1)$ and hence its error rate dominates. The power allocation algorithm tries to reduce the BLER by allocating more power to the $1^{\text {st }}$ stream and thus reducing the $1^{\text {st }}$ step BER.

SNR gain of optimization: Comparing the optimized average BLER to non-optimized one in Fig. 2, one concludes that at high SNR the optimization brings about $3 \mathrm{~dB}$ SNR gain (for $m=2$ ). This can be explained by the following simple argument. The average BLER of the optimized system can be bounded as follows,

$$
\bar{P}_{B}\left(\alpha_{1}=m, \alpha_{2}^{o p t}, \ldots, \alpha_{m}^{o p t}\right) \leq \bar{P}_{B}\left(\boldsymbol{\alpha}^{o p t}\right) \leq \bar{P}_{B}\left(\alpha_{1}=\ldots=\alpha_{m}=1\right)(20)
$$
where $\boldsymbol{\alpha}^{\text {opt }}$ is the optimum power allocation vector. The upper bound is the non-optimized BLER and the lower bound is the optimized BLER for which $\alpha_{1}$ is increased to $m$ (which cannot increase the BLER). Additionally, since $\bar{P}_{B} \geq \bar{P}_{e 1}$,

$$
\bar{P}_{e 1}\left(\alpha_{1}=m\right) \leq \bar{P}_{B}\left(\alpha_{1}=m, \alpha_{2}^{o p t}, \ldots, \alpha_{m}^{o p t}\right)
$$

Substituting (7) and (21) in (20), one obtains 


$$
\bar{P}_{e 1}\left(\alpha_{1}=m\right) \leq \bar{P}_{B}\left(\boldsymbol{\alpha}^{o p t}\right) \leq \bar{P}_{B}\left(\alpha_{1}=\ldots=\alpha_{m}=1\right) \approx \bar{P}_{e 1}\left(\alpha_{1}=1\right),
$$

from which one concludes the SNR gain of the optimization is bounded as

$$
\Delta \gamma \leq m
$$

Since the lower bound in (22) is tight at high SNR,

$$
\Delta \gamma \approx m \text { for } \gamma_{0} \gg 1
$$

It should be noted that this conclusion hold true for any modulation format. It also holds true if the power allocation is optimized instantaneously rather than on average, as the optimized system cannot perform better than a hypothetical one for which all the power is allocated to the $1^{\text {st }}$ Tx and an additional power source is used to supply power to the other Txs to keep their BER low enough.

For $m=2$, the SNR gain of the optimum power allocation is roughly the same, at high SNR, as that of the optimal ordering procedure (see [5] for details). The computational complexity, however, of the former is much less than that of the latter. Hence, the average power optimization can be used instead of the optimal ordering with the same performance (in terms of the average BLER/TBER).

Average vs. instantaneous optimization: Since the average power allocation achieves the upper bound in (23) and since the instantaneous allocation cannot perform worse than the average one, one concludes that the average and instantaneous optimizations are equivalent (in terms of average BLER) at high SNR. Clearly, the average allocation is preferable to use as its complexity is much less both in terms of computations and the feedback channel required (only one computation of $\boldsymbol{\alpha}^{o p t}$ is required as long as $\gamma_{0}$ stays the same and only $\gamma_{0}$ needs to be fed back to the Tx end) compared to instantaneous one (each channel instant requires its own optimization and feedback session). As the detailed analysis below demonstrates, these conclusions also hold if TBER is used as an optimization criterion.

Optimum power allocation using average TBER: In a similar way, the average TBER can be used (in (15)) as a goal function in the Lagrange multiplier technique to find the optimum power allocation. Numerical analysis demonstrates that, for $m=2$, the TBER is convex in $\alpha$ and hence the optimum solution is unique for arbitrary SNR. This solution can be found numerically, and for high-SNR mode an approximate (but accurate) closed-form analytical solution can be obtained using the Newton-Raphson method. In this mode, the average TBER (assuming BPSK modulation) can be approximated as (a proof is omitted due to the page limit),

$$
\bar{P}_{e t}(\boldsymbol{\alpha}) \approx \sum_{i=1}^{m} \frac{\beta_{i}}{\left(\alpha_{i} \gamma_{0}\right)^{n-m+i}}, \beta_{i}=\frac{3 C_{2 i-1}^{i}}{4^{n-m+i}} \frac{m-i+2}{2 m}
$$

Since $\bar{P}_{e t}(\boldsymbol{\alpha})$ is convex (because each term in the sum is convex), the solution to the optimization problem is the unique global minimum. For the case of $m=2$, the solution is particularly simple:

$$
\alpha_{1} \approx 2\left(1-\frac{c}{(n+1) \sqrt[n+1]{\gamma_{0}}}\right)^{n+1}, \alpha_{2}=2-\alpha_{1}, c=\sqrt[n+1]{\frac{n}{4(n-1)}}
$$

Clearly, this power allocation is close to that in (19), which is also obvious from Fig. 1 and hence the choice of the optimization criteria (either BLER or TBER) does not affect significantly the final result. This is not a surprise as the $1^{\text {st }}$ step error rate is dominant (due to the lowest diversity order) in terms of both the average BLER and TBER and hence most of the total Tx power goes to the $1^{\text {st }} \mathrm{Tx}$.

Optimum power allocation using instantaneous BLER/TBER: Similarly to the average power optimization above, the instantaneous power can be optimally allocated using either BLER or TBER as the optimization criteria. Since an analytical solution is challenging, a numerical technique can be used. The optimum allocation in terms of BLER is unique for any modulation whose BER can be represented as linear combination (with non-negative coefficients) of $Q(\sqrt{a \gamma})$ and/or $\exp (-b \gamma)$ (this follows directly from the proof above in terms of the average BLER, by observing that the averaging does not affect the argument). The uniqueness of the TBER-based optimum power allocation is an open problem (numerical evidence suggests that this allocation is unique).

Fig. 2 compares the average BER of instantaneous and average power optimization. Clearly, the results are quite close to each other, especially for $\gamma_{0} \geq 20 d B$. Essential difference between these two is that the former achieves the maximum SNR gain (as in (24)) for smaller SNR (and, of course, the instantaneous optimization performs better in terms of instantaneous BER, especially for some channel realizations that do not favor the average power allocation). Our main conclusion here is that the average power optimization can be used instead of instantaneous one at high SNR without any visible BER penalty but with much less complexity.

\section{REFERENCES}

[1] G.J. Foschini et al, Analysis and Performance of Some Basic Space-Time Architectures, IEEE Journal Selected Areas Comm., v. 21, N. 3, pp. 281-320, April 2003.

[2] G.J Foschini et al, Simplified Processing for High Spectral Efficiency Wireless Communication Employing Multi-Element Arrays, IEEE Journal on Selected Areas in Communications, v. 17, N. 11, pp. 1841-1852, Nov. 1999.

[3] H. Zhu et al, An Improved Square-Root Algorithm for BLAST, IEEE Sig. Proc. Letters, v. 11, N. 9, pp. 772-775, Sep. 2004.

[4] S.H. Nam et al, Transmit Power Allocation for a Modified V-BLAST System, IEEE Trans. Comm., v. 52, N. 7, pp.1074-1079, Jul. 2004.

[5] S. Loyka, F. Gagnon, Performance Analysis of the VBLAST Algorithm: an Analytical Approach, IEEE Trans. Wireless Comm., v. 3, No. 4, pp. 1326-1337, July 2004.

[6] S. Loyka, V-BLAST in a Rayleigh Fading Channel: Analytical Outage and BER Analysis, Seminar Notes, ETH Zurich, Feb. 162005.

[7] S. Loyka, F. Gagnon, V-BLAST without Optimal Ordering: Analytical Performance Evaluation for Rayleigh Fading Channels, IEEE Trans. Communications, under review

[8] S. Boyd, L. Vandenberghe, Convex Optimization, Cambridge University Press, 2004. 